\title{
The tumor suppressor PTEN interacts with p53 in hereditary cancer (Review)
}

\author{
ATSUKO NAKANISHI ${ }^{*}$, YASUKO KITAGISHI ${ }^{*}$, YASUNORI OGURA and SATORU MATSUDA* \\ Department of Food Science and Nutrition, Nara Women's University, Kita-Uoya Nishimachi, Nara 630-8506, Japan
}

Received January 30, 2014; Accepted March 26, 2014

DOI: 10.3892/ijo.2014.2377

\begin{abstract}
Numerous hereditary syndromes caused by mutations in multiple tumor suppressor genes can cause cancers. Germline mutations in PTEN and $p 53$ tumor suppressor cause Cowden syndrome and Li-Fraumeni syndrome, respectively. There exists some phenotypic overlap in these syndromes, and they are associated with high risks of breast cancer. The tumor suppressor protein PTEN is a dual-specificity phosphatase which has protein phosphatase activity and lipid phosphatase activity that antagonizes PI3K activity. Cells that lack PTEN have constitutively higher levels of PIP3 and activated downstream targets. PTEN gene is recognized as one of the most frequently mutated or mutated in many human cancers. Li-Fraumeni syndrome results from germline mutations of the tumor suppressor p53 gene encoding a transcriptional factor able to regulate cell cycle and apoptosis when DNA damage occurs. The p53 protein cooperates with PTEN and might be an essential blockage in development of mammary tumors. Many findings have demonstrated that PTEN as well as p53 plays a critical role in DNA damage response. This review summarizes the function of PTEN and p53 in carcinogenic cell signaling. In addition, we will discuss the role of PTEN signaling through its interaction with $\mathrm{p} 53$ and MDM2 pathways for the potential implications in hereditary cancer prevention and therapeutic intervention.
\end{abstract}

Correspondence to: Professor Satoru Matsuda, Department of Food Science and Nutrition, Nara Women's University, Kita-Uoya Nishimachi, Nara 630-8506, Japan

E-mail: smatsuda@cc.nara-wu.ac.jp

*Contributed equally

Abbreviations: ATF2: activating transcription factor 2; NF- $\kappa \mathrm{B}$, nuclear factor $\kappa \mathrm{B}$; MDM2, murine double minute 2; PTEN, phosphatase and tensin homologue deleted on chromosome 10; PIP3, phosphatidylinositol 3,4,5-triphosphate; PIP2, phosphatidylinositol 4,5-bisphosphate; PI3K, phosphoinositide-3 kinase; PTP, protein tyrosine phosphatase; PPAR, peroxisome proliferator-activated receptor; TGF, transforming growth factor

Key words: hereditary cancer,PTEN, p53, MDM2, protein interaction, protein degradation, cell signaling

\section{Contents}

1. Introduction

2. Expression, structure and characteristics of PTEN and p53

3. Protein interaction and functional interplay between PTEN and $\mathrm{p} 53$

4. Involvement of PTEN and p53 tumor suppressors in hereditary cancer

5. Perspective

\section{Introduction}

PTEN (phosphatase and tensin homolog deleted in chromosome 10) is a tumor suppressor gene that is deleted or mutated in a variety of human cancers $(1,2)$. Germ line mutations of PTEN are also the cause of PTEN hamartoma tumor syndromes (Cowden syndrome, Bannayan-Riley-Ruvalcaba syndrome, PTEN-related Proteus syndrome, Proteus-like syndrome) with increased risk for the development of cancers (3). Cowden syndrome is a rare, autosomal dominant, familial cancer syndrome characterised by hamartomas, acral keratosis, multiple smooth facial papules, and multiple oral papillomas (4). In contrast, Bannayan-Riley-Ruvalcaba syndrome is characterized by lipomatosis, macrocephaly, hemangiomatosis (5). Loss of heterozygosity (LOH) studies suggest that PTEN may play its most important role in advanced cancers of particular tissue (6). Alterations of PTEN in tumors are associated with a poor prognosis (7). Germinal mutations of the $p 53$ gene constitute an etiological genetic base of Li-Fraumeni syndrome, which is a rare genetically and clinically heterogeneous autosomal dominant inherited cancer disorder, characterized by a specific range of tumors observed at an early age, in particular a predominance of bone and soft tissue sarcomas and breast cancer (8). Generally, cells with dysfunctional p53 are predisposed to development of cancer phenotype. Of importance, it is mutated frequently in the common human malignancies of the breast and colon rectum and also in other significant cancers such as glioblastoma with less frequency (9).

The PTEN and $p 53$ tumor suppressors are among the most commonly inactivated or mutated genes in human cancer (10). The PTEN has been shown to be involved in a complex network of interactions with p53 (Fig. 1). Although they are functionally distinct, reciprocal cooperation has been proposed, as PTEN 
is thought to regulate p53 stability, and p53 to enhance PTEN transcription. Once PTEN is lost, however, the p53 pathway is strongly activated $(11,12)$. Furthermore, an absence of PTEN cooperates with an absence of p53 to promote cancer (13). The inactivation of tumor suppression may be caused by lack of key interaction partners. Recent studies have revealed a functional ubiquitin ligase for tumor suppressors playing a pivotal role in tumor cell survival $(14,15)$. Mutations found in genes such as p53 and PTEN have emerged as high penetrance susceptibility genes and are clinically relevant for determination of cancer risk. In this review, we summarize the current research and our view of how and when PTEN and p53 with their partners transduce signals downstream and the implications for cancerassociated biology.

\section{Expression, structure and characteristics of PTEN and p53}

The human genomic PTEN locus consists of 9 exons on chromosome 10q23.3 encoding a $5.5 \mathrm{~kb}$ mRNA that specifies a 403 amino acid open reading frame $(16,17)$. The translation product is a $53 \mathrm{kDa}$ protein with homology to tensin and protein tyrosine phosphatases. PTEN is ubiquitously expressed throughout early embryogenesis in mammals (18). PTEN gene can be upregulated by early growth regulated transcription factor 1 , peroxisome proliferator activated receptor $\gamma(\operatorname{PPAR} \gamma), \mathrm{p} 53$, and activating transcription factor 2 (ATF2) (19-22), while transforming growth factor (TGF)- $\beta$, nuclear factor $\kappa \mathrm{B}(\mathrm{NF}-\kappa \mathrm{B})$, and Jun negatively regulate PTEN expression (23-25). Of note, rosemary extract represses $P T E N$ expression in K562 leukemic culture cells (26). PTEN activity can be regulated by posttranslational regulation including phosphorylation, acetylation, and oxidation. Methylation of the PTEN promoter can result in transcriptional silencing of the PTEN gene (27). Schematic structure of the predicted PTEN protein is shown in Fig. 2. PTEN negatively regulates the activity of PI3K/AKT signaling through converting phosphatidylinositol 3,4,5-triphosphate (PIP3) into phosphatidylinositol 4,5- bisphosphate (PIP2). The PIP3 is the principal second messenger of the PI3K pathway that mediates receptor tyrosine kinase (RTK) signaling to the survival kinase AKT. Activated AKT transfers a phosphate group to target proteins involved in cell survival, cell cycling, proliferation, and migration, which also are all critical for tumor development $(28,29)$.

PTEN acts as regulator of maintaining basal levels of PIP3 below a threshold for the signaling activation. PTEN protein consists of $\mathrm{N}$-terminal phosphatase, and $\mathrm{C}$-terminal $\mathrm{C} 2$, and PDZ (PSD-95, DLG1, and ZO-1) binding domains. The PTEN CX5R(S/T) motif resides within an active site that surrounds the catalytic signature with three basic residues, which are critical for PTEN lipid phosphatase activity. The structure endows PTEN with its preference for acidic phospholipid substrates such as PIP3. Overexpression of PTEN induces growth suppression by promoting cell cycle arrest, which requires lipid phosphatase activity $(30,31)$. Overexpression of PTEN also correlates with decreased levels and nuclear localization of cyclin D1 (32), a key cell cycle molecule regulated by AKT. One mechanism by which PTEN induces cell cycle arrest is the regulation of AKT activity such that levels of the cell cycle inhibitor p27kip1 are increased (33). However, despite the main role of PTEN as a negative regulator of the PI3K pathway, studies report various tumor suppressive activities for PTEN that are exerted from within the nucleus, where catalysis of PIP3 does not seem to represent a dominant function of this enzyme (34). The nuclear PTEN activities may include the regulation of genomic stability, cell cycle progression, and gene expression.

The p53 gene, located on chromosome 17p 13.1 and encoding a nuclear 393-amino acid protein, acts to control cell growth and apoptosis (Fig. 2). The p53 protein is a transcription factor which is able to induce G1 arrest of the cell cycle by transactivating several downstream genes. Inactivation of p53 gene is a common event in the development of most types of cancer. The importance of p53 as an inherited cancer susceptibility gene has been demonstrated in Li-Fraumeni syndrome (35). However, there are still a significant number of Li-Fraumeni families for which no underlying genetic determinant has been identified. It would be beneficial to understand the alterations of genes or additional components involved in DNA damage recognition, DNA repair, and/or cell cycle checkpoint pathways responsible for the specific phenotype.

\section{Protein interaction and functional interplay between PTEN and p53}

PTEN and p53 is known to interact and regulate each other at the transcription as well as protein level, which could be at the important control machinery for switching between survival and death. This cross talk is frequently a combination of reciprocally antagonistic pathways, which often involves another tumor suppressor gene MDM2, and may serve as an added regulatory effect on the expression of key genes involved in cancer. It has also been revealed that PTEN regulates p53 stability and in turn regulates its own transcriptional activity.

At transcription level. The PTEN and p53 complex enhances p53 DNA binding and transcriptional activity (36). An important p53 function is to act as a transcription factor by binding to the specific DNA consensus sequence in responsive genes, which may increase the synthesis of p21waf1 that is an important protein involved in cell cycle arrest (37). In addition, one of transcriptional targets of p53 is PTEN. One way by which p53 inhibits production of PIP3 indirectly is by inducing the expression of PTEN (38). Under hypoxic conditions PTEN and p53 form a complex in the nucleus and induce strong expression of the tumor suppressor Maspin (39). Loss of PTEN attenuates the induction of Maspin even in the presence of wild-type p53. The integration of PTEN and p53 into a common pathway for the induction of Maspin may constitute a tumor suppressor network (40).

MDM2 is an oncoprotein that controls tumorigenesis, its mRNA level is transcriptionally regulated by $\mathrm{p} 53$ in response to DNA damage such as oxidative stress (41). The MDM2 protein and subcellular localization are post-translationally modulated by AKT (42). PTEN inhibits PI3K/AKT signaling that promotes translocation of MDM2 into the nucleus. In addition, PTEN modulates $M D M 2$ transcription and isoform selection by negatively regulating its promoter (43). In PTEN-null cells, MDM2 promoter activity is upregulated, resulting in increased $M D M 2$ expression. Furthermore, PTEN controls MDM2 promoter activity through its lipid phosphatase activity, independent 


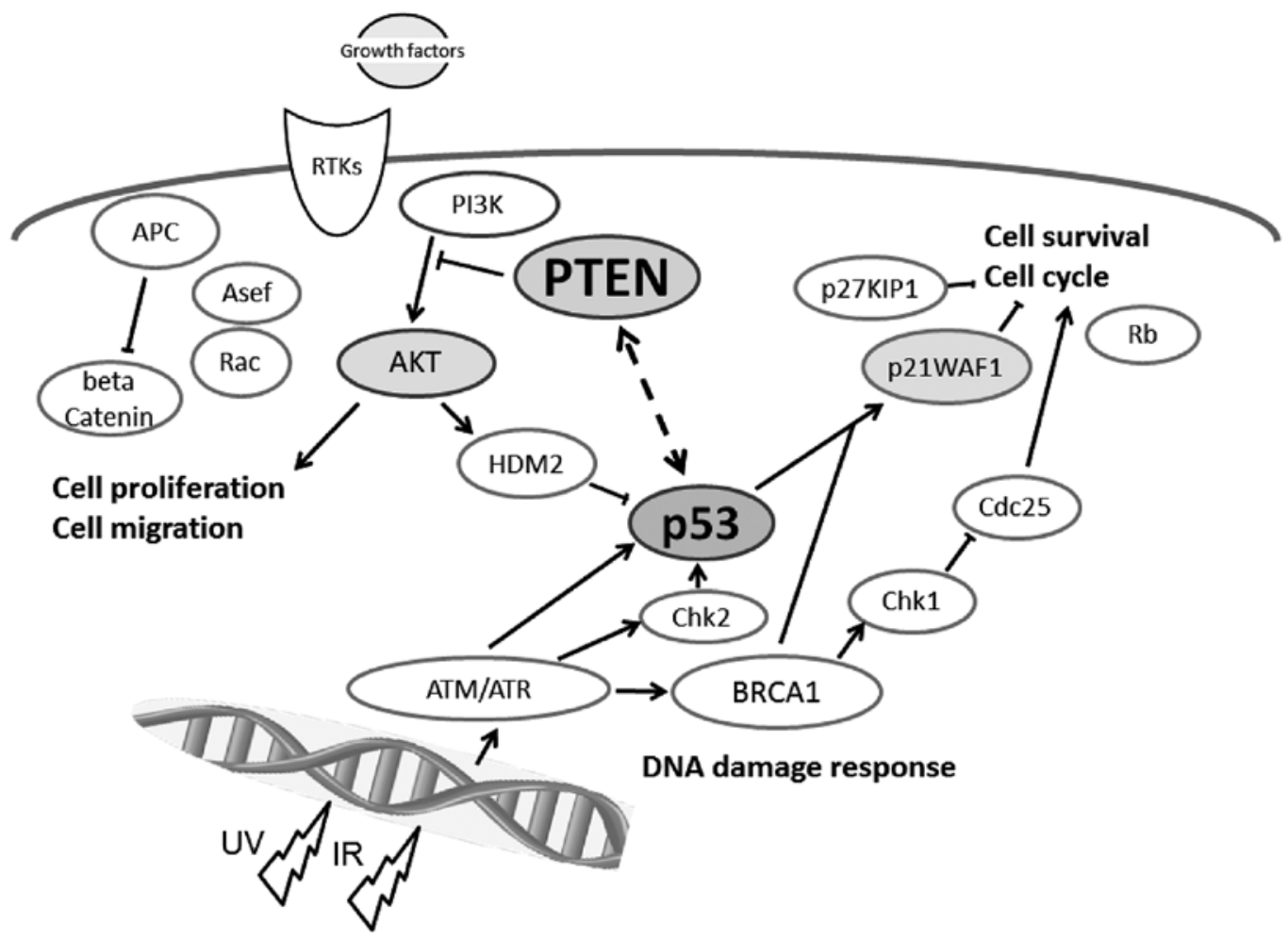

Figure 1. The proposed integrative model of tumor suppressor signaling including PTEN and p53. Examples of molecules known to act on DNA damage response, cell proliferation, and cell cycle via the regulatory pathways are shown. Note that some critical pathways have been omitted for clarity.
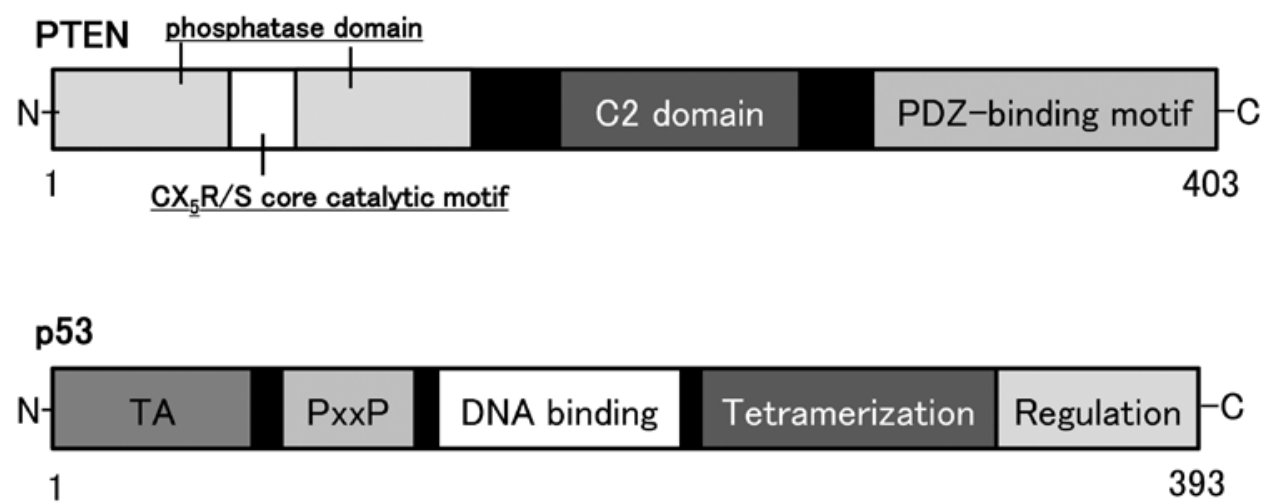

Figure 2. The structures of PTEN and p53 protein. The predicted consensual domain structures for each protein are depicted. The functionally important sites are also shown. TA, transactivation domain; PxxP, proline rich region; $\mathrm{C} 2$ domain, a protein structural domain involved in targeting proteins to cell membranes; PDZ, a common structural domain in signaling proteins (PSD95, Dlg and ZO-1).

of p53 (36). Although another transcription factors such as AP-1 are able to modulate $M D M 2$ transcription, they have been characterized to work through the p53 responsible promoter (44). MDM2 is a key regulator of p53. It regulates the activity of $\mathrm{p} 53$ protein by blocking its transcriptional activity, exporting nuclear p53 protein into the cytoplasm, and/or by promoting the degradation of the $\mathrm{p} 53$ protein. PTEN upregulates the p53 level as well as its activity by downregulating MDM2 transcription and p53 binding activity (45). However, in the absence of p53, PTEN may have a role inhibiting MDM2-mediated carcinogenesis through regulation of MDM2 transcription as well as the isoform selection. MDM2 is degraded, when restricted to the cytoplasm. The ability of PTEN to inhibit the nuclear entry of MDM2 increases the cellular content and transactivation of the p53 to promote the induction of responsive genes such as p21 (46).

Protein modification and interaction. p53 and AKT influence the process of apoptosis in opposite ways. The AKT promotes cell survival by suppressing pro-apoptotic proteins such as Bad through phosphorylation (47). There is cross talk between p53 and AKT involving gene transcription as well as posttranslational protein modifications. p53 inhibits PIP3 production indirectly by repressing the catalytic subunit of PI3K. A subsequent p53-induced expression of PTEN causes the p53-PTEN interaction, which then suppresses the cell survival machinery of AKT pathway. AKT phosphorylates MDM2 to translocate into the nucleus (48). In addition, PTEN physically associates with endogenous p53 and regulates 


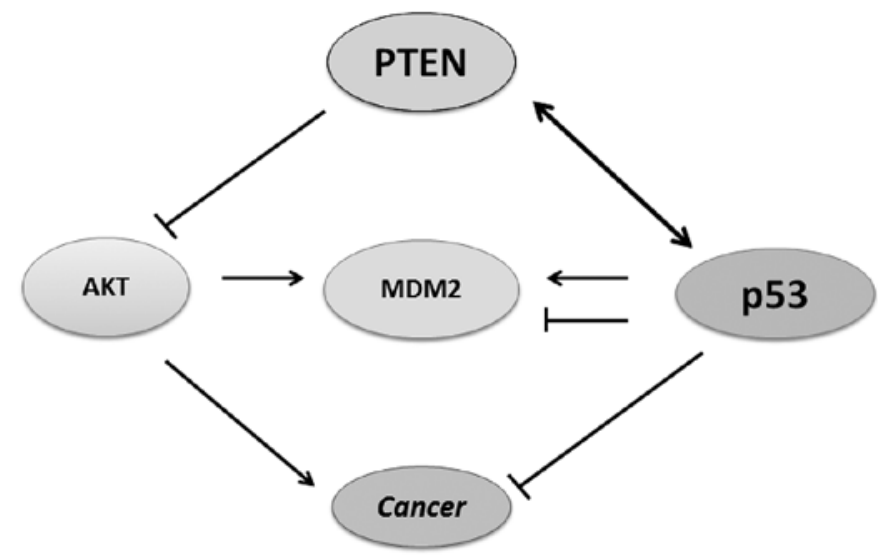

Figure 3. Implication of various feedback loops involving the PTEN-AKTp53-MDM2 regulatory network. Interactions are shown as arrows indicating activation, while hammerheads indicate inhibition. Note that some critical pathways have been omitted for clarity.

the transcriptional activity of p53 by modulating its DNA binding (36). PTEN is required for the maintenance of $\mathrm{p} 53$ acetylation, which is also required for target gene transcription (49).

Growth factor-activated AKT signaling promotes progression of cell cycles by acting on downstream factors involved in controlling the G1/S and/or G2/M transitions. Several studies have also implicated AKT in modulating DNA damage responses and genome stability (50). AKT therefore modifies downstream signaling in complex ways. In addition, PTEN also plays a critical role in DNA damage repair and DNA damage response through its interaction with p53 pathways in an AKT-independent manner (51). Furthermore, nuclear PTEN is sufficient to reduce tumor progression in a p53-dependent manner. It has also been suggested that nuclear PTEN plays a unique role to protect cells upon oxidative damage and to regulate carcinogenesis (52), thus, the PTEN-p53-MDM2-AKT loop becomes dominant (Fig. 3).

Protein degradation. One aspect of the PTEN tumor suppressor signaling is achieved through stabilization of the p53 protein. PTEN has been shown to physically interact with p53 and prevent its degradation by excluding a portion of p53 protein from the p53 and MDM2 complex. Evidence indicates the existence of a link between PTEN and p53 functions through the control of the phosphorylation state of MDM2. AKT mediates MDM2 nuclear translocation by its phosphorylation. In the nucleus, MDM2 negatively regulates p53 by binding and signaling for destabilization (53). Therefore, attenuation of the AKT pathway by PTEN protects p53 from MDM2 mediated degradation and inactivation. The p53 and MDM2 complex is transported from the nucleus into the cytoplasm where MDM2 serves as an E3 ubiquitin ligase (54). Consequently, p53 and MDM2 form a regulatory feedback loop in which p53 positively regulates MDM2 expression, whereas MDM2 negatively regulates the level of p53 protein. Thus, PTEN may protect p53 from MDM2-mediated degradation, whereas p53 can enhance the transcription of PTEN (Fig. 3). Therefore, inactivation of either gene result in lower protein levels of the other gene.
The instability of PTEN correlated with its missense mutations has been shown to involve protein interactions. PTEN may be regulated by ubiquitin-mediated proteasomal degradation, a common mechanism to control protein levels. In cells, a ubiquitin ligase NEDD4-1 negatively regulates PTEN stability by catalyzing PTEN ubiquitination (55). As truncation or mutation of the $\mathrm{C} 2$ domain of PTEN makes the protein unstable and accelerates protein degradation, the $\mathrm{C} 2$ domain seems to regulate the phosphatase domain through maintaining the protein stability (56). In addition to the $\mathrm{C} 2$ domain, the C-terminus of PTEN contains two PEST (proline, glutamic acid, serine and threonine) sequences involved in ubiquitin protein degradation pathway. Treatment of cells with proteasome inhibitors can cause an increase of PTEN protein level $(57,58)$. Several NEDD4-like E3 also regulate p53. Interestingly, multiple NEDD4-like E3 show ligase independent function. Furthermore, most of NEDD4-like E3 are frequently regulated by phosphorylation, ubiquitination, translocation, and transcription in cancer cells. NEDD4-like ubiquitin protein ligase-1 (NEDL1) is a type E3 ubiquitin protein ligase. Functional interaction of NEDL1 with p53 might contribute to the induction of apoptosis in cancer cells bearing wild-type p53 $(59,60)$.

Casein kinase II-mediated phosphorylation stabilizes the PTEN protein by preventing its proteasomal degradation, while keeping it in an inactive state. Inhibition of the PTEN phosphorylation by the Casein kinase II results in increased PTEN activity and a corresponding reduction in AKT activation (61). Importantly, inhibitors of Casein kinase II also activate p53 function in wild-type, but not in p53 mutant cells. Activation of p53 function is involved in increased DNA-binding ability, transcriptional activation, increased expression of p53 target genes, associated with cell cycle progression and apoptosis. In addition, inhibitors of Casein kinase II increase senescence p53-dependently (62), thus, Casein kinase II may control the PTEN and the $\mathrm{p} 53$ balance.

\section{Involvement of PTEN and p53 tumor suppressors in hereditary cancer}

The PTEN gene is found in $80 \%$ of Cowden syndrome patients $(63,64)$. Mutations of the PTEN gene are thus frequent in hereditary cancer syndromes, and are found in all exons except 1, 4, and 9 in Cowden syndrome (63-65). These mutations target the PTEN gene not only at its coding regions, but also at exon-intron boundaries and promoter regions, most of which have a major impact in the PTEN protein levels of expression, being causative of PTEN functional deficiency and considered as pathogenic $(66,67)$. It has been suggested that the differential expression of the PTEN gene correlates with the different phenotypes. Mutations targeting the PTEN coding region include frame-shift and nonsense mutations, which also generate unstable truncated PTEN proteins, as well as missense mutations that result in individual amino acid changes. Functional analyses of these missense mutations have revealed that the amino acid substitutions generate PTEN proteins with impaired intrinsic catalytic activity and/ or protein stability (68-70).

Some examples have been described of missense PTEN mutations that do not affect the intrinsic catalysis nor the 
stability of the protein, but rather impair essential regulatory PTEN properties, such as binding to membranes or nuclear entry (71). PTEN hamartoma tumor syndrome is the term used to describe Cowden syndrome (65). Mutations in PTEN together with p53 make the tumor suppressor genes one of the most frequently affected in human malignancies in solid tumors (72-74). PTEN is considered haplo-insufficient to prevent certain malignancies, suggesting that dosage is important for its function, as it is influenced by a given point mutation in the catalytic activity of the enzyme (75). Tumors initiated by a subtle downregulation of a tumor suppressor gene can progress in the absence of $\mathrm{LOH}$ of the wild-type allele (76). These regulatory cues are presumed to play a key role in tumorigenesis through the alteration of the appropriate levels, localization, and activity of PTEN. However, it has been shown that PTEN germ-line SNPs are unlikely to have an important role in hereditary prostate susceptibility (77). The lifetime risk of breast cancer for Cowden syndrome patients is $81 \%$ (78), and bilateral risk-reducing mastectomy with immediate reconstruction is performed to eliminate further risk of breast cancer (79).

\section{Perspective}

Advances in the field of hereditary cancer genetics have led to an improved understanding of detection and prevention strategies. Germline genetic testing for mutations in PTEN and p53 allows for the identification of individuals at increased risk for breast, ovarian and other cancers. PTEN and p53 may be regulated and interact with each other at multiple levels including transcription, protein modulation, and protein stability $(80,81)$. Understanding the regulation is crucial for the effective design of novel cancer therapeutics. In addition, it is important to investigate the functional linkage between PTEN, p53 and MDM2 isoforms in human cancer samples, and elucidation of interaction-specific functions may provide insight into regulatory aspects of these tumor suppressors as well as opportunities for therapeutic intervention. Further mechanistic studies are needed in order to understand the precise molecular mechanisms for the effective treatment of both cancer and other diseases with their alteration.

\section{Acknowledgements}

This work was supported by grants-in-aid from the Ministry of Education, Culture, Sports, Science and Technology in Japan. In addition, this work was supported in part by the grant from SHIN-EI Pharmaceutical Co., Ltd., and the grant from Nakagawa Masashichi Shoten Co., Ltd.

\section{References}

1. Merritt MA and Cramer DW: Molecular pathogenesis of endometrial and ovarian cancer. Cancer Biomark 9: 287-305, 2010.

2. Song MS, Salmena L and Pandolfi PP: The functions and regulation of the PTEN tumour suppressor. Nat Rev Mol Cell Biol 13: 283-296, 2012

3. Hobert JA and Eng C: PTEN hamartoma tumor syndrome: an overview. Genet Med 11: 687-694, 2009.

4. Conti S, Condò M, Posar A, et al: Phosphatase and tensin homolog (PTEN) gene mutations and autism: literature review and a case report of a patient with Cowden syndrome, autistic disorder, and epilepsy. J Child Neurol 27: 392-397, 2012.
5. Litzendorf M, Hoang K and Vaccaro P: Recurrent and extensive vascular malformations in a patient with Bannayan-RileyRuvalcaba syndrome. Ann Vasc Surg 25: 1138.e15-9, 2011.

6. Morgan TM, Koreckij TD and Corey E: Targeted therapy for advanced prostate cancer: inhibition of the PI3K/Akt/mTOR pathway. Curr Cancer Drug Targets 9: 237-249, 2009.

7. Knobbe CB, Merlo A and Reifenberger G: Pten signaling in gliomas. Neuro Oncol 4: 196-211, 2002.

8. Li FP, Fraumeni JF Jr, Mulvihill JJ, et al: A cancer family syndrome in twenty-four kindreds. Cancer Res 48: 5358-5362, 1988.

9. Masui K, Cloughesy TF and Mischel PS: Review: molecular pathology in adult high-grade gliomas: from molecular diagnostics to target therapies. Neuropathol Appl Neurobiol 38: 271-291, 2012.

10. Dong JT: Prevalent mutations in prostate cancer. J Cell Biochem 97: 433-447, 2006.

11. Chen Z, Trotman LC, Shaffer D, et al: Crucial role of p53-dependent cellular senescence in suppression of Ptendeficient tumorigenesis. Nature 436: 725-730, 2005.

12. Kim J, Eltoum IE, Roh M, Wang J and Abdulkadir SA: Interactions between cells with distinct mutations in c-MYC and Pten in prostate cancer. PLoS Genet 5: e1000542, 2009.

13. Blanco-Aparicio C, Renner O, Leal JF and Carnero A: PTEN, more than the AKT pathway. Carcinogenesis 28: 1379-1386, 2007.

14. Faesen AC, Dirac AM, Shanmugham A, Ovaa H, Perrakis A and Sixma TK: Mechanism of USP7/HAUSP activation by its C-terminal ubiquitin-like domain and allosteric regulation by GMP-synthetase. Mol Cell 44: 147-159, 2011.

15. Sacco JJ, Coulson JM, Clague MJ and Urbé S: Emerging roles of deubiquitinases in cancer-associated pathways. IUBMB Life 62: 140-157, 2010.

16. Okumura N, Yoshida H, Kitagishi Y, Murakami M, Nishimura Y and Matsuda S: PI3K/AKT/PTEN signaling as a molecular target in leukemia angiogenesis. Adv Hematol 2012: 843085, 2012.

17. Okumura N, Yoshida H, Kitagishi Y, Nishimura Y and Matsuda S: Alternative splicings on p53, BRCA1 and PTEN genes involved in breast cancer. Biochem Biophys Res Commun 413: 395-399, 2011.

18. Croushore JA, Blasiole B, Riddle RC, et al: Pten a and pten b genes play distinct roles in zebrafish embryogenesis. Dev Dyn 234: 911-921, 2005.

19. Teresi RE, Shaiu CW, Chen CS, Chatterjee VK, Waite KA and Eng C: Increased PTEN expression due to transcriptional activation of PPARgamma by Lovastatin and Rosiglitazone. Int J Cancer 118: 2390-2398, 2006.

20. Shen YH, Zhang L, Gan Y, et al: Up-regulation of PTEN (phosphatase and tensin homolog deleted on chromosome ten) mediates p38 MAPK stress signal-induced inhibition of insulin signaling. A cross-talk between stress signaling and insulin signaling in resistin-treated human endothelial cells. J Biol Chem 281: 7727-7736, 2006.

21. Pan L, Lu J, Wang X, et al: Histone deacetylase inhibitor trichostatin a potentiates doxorubicin-induced apoptosis by up-regulating PTEN expression. Cancer 109: 1676-1688, 2007.

22. Lee YR, Yu HN, Noh EM, et al: Peroxisome proliferator-activated receptor gamma and retinoic acid receptor synergistically up-regulate the tumor suppressor PTEN in human promyeloid leukemia cells. Int J Hematol 85: 231-237, 2007.

23. Yang Y, Zhou F, Fang Z, et al: Post-transcriptional and posttranslational regulation of PTEN by transforming growth factor-beta1. Cell Biochem 106: 1102-1112, 2009.

24. Han S, Ritzenthaler JD, Zheng Y and Roman J: PPARbeta/delta agonist stimulates human lung carcinoma cell growth through inhibition of PTEN expression: the involvement of PI3K and NF-kappaB signals. Am J Physiol Lung Cell Mol Physiol 294: L1238-L1249, 2008.

25. Vasudevan KM, Burikhanov R, Goswami A and Rangnekar VM: Suppression of PTEN expression is essential for antiapoptosis and cellular transformation by oncogenic Ras. Cancer Res 67: 10343-10350, 2007.

26. Yoshida H, Okumura N, Kitagishi Y, Nishimura Y and Matsuda S: Ethanol extract of rosemary repressed PTEN expression in K562 culture cells. Int J Appl Boil Pharm Technol 2: 316-322, 2011.

27. Mueller S, Phillips J, Onar-Thomas A, et al: PTEN promoter methylation and activation of the PI3K/Akt/mTOR pathway in pediatric gliomas and influence on clinical outcome. Neuro Oncol 14: 1146-1152, 2012. 
28. Faurschou A, Gniadecki R, Calay D and Wulf HC: TNF-alpha impairs the $\mathrm{S}-\mathrm{G} 2 / \mathrm{M}$ cell cycle checkpoint and cyclobutane pyrimidine dimer repair in premalignant skin cells: role of the PI3K-Akt pathway. J Invest Dermatol 128: 2069-2077, 2008.

29. Chen Y, Wang BC and Xiao Y: PI3K: a potential therapeutic target for cancer. J Cell Physiol 227: 2818-2821, 2012.

30. Choi Y,Zhang J, Murga C, et al: PTEN, but not SHIP and SHIP2, suppresses the PI3K/Akt pathway and induces growth inhibition and apoptosis of myeloma cells. Oncogene 21: 5289-5300, 2002.

31. Petrella BL and Brinckerhoff CE: PTEN suppression of YY1 induces HIF-2 activity in von-Hippel-Lindau-null renal-cell carcinoma. Cancer Biol Ther 8: 1389-1401, 2009.

32. Yamamoto M, Tamakawa S, Yoshie M, Yaginuma $Y$ and Ogawa K: Neoplastic hepatocyte growth associated with cyclin D1 redistribution from the cytoplasm to the nucleus in mouse hepatocarcinogenesis. Mol Carcinog 45: 901-913, 2006.

33. Andrés-Pons A, Gil A, Oliver MD, Sotelo NS and Pulido R: Cytoplasmic p27Kip1 counteracts the pro-apoptotic function of the open conformation of PTEN by retention and destabilization of PTEN outside of the nucleus. Cell Signal 24: 577-587, 2012.

34. Planchon SM, Waite KA and Eng C: The nuclear affairs of PTEN. J Cell Sci 121: 249-253, 2008

35. Palmero EI, Achatz MI, Ashton-Prolla P, Olivier M and Hainaut P: Tumor protein 53 mutations and inherited cancer: beyond Li-Fraumeni syndrome. Curr Opin Oncol 22: 64-69, 2010.

36. Freeman DJ, Li AG, Wei G, et al: PTEN tumor suppressor regulates 533 protein levels and activity through phosphatasedependent and -independent mechanisms. Cancer Cell 3: 117-130, 2003.

37. Lo PK, Lee JS and Sukumar S: The p53-p21WAF1 checkpoint pathway plays a protective role in preventing DNA rereplication induced by abrogation of FOXF1 function. Cell Signal 24: 316-324, 2012.

38. Puszyński K, Hat B and Lipniacki T: Oscillations and bistability in the stochastic model of p53 regulation. J Theor Biol 254: 452-465, 2008

39. Eitel JA, Bijangi-Vishehsaraei K, Saadatzadeh MR, et al: EN and p53 are required for hypoxia induced expression of maspin in glioblastoma cells. Cell Cycle 8: 896-901, 2009.

40. Zhang M: PTEN in action: coordinating with $\mathrm{p} 53$ to regulate maspin gene expression. Cell Cycle 8: 1112-1113, 2009.

41. Wang $X$ and Jiang X: Mdm2 and MdmX partner to regulate p53. FEBS Lett 586: 1390-1396, 2012.

42. Levav-Cohen $Y$, Haupt S and Haupt Y: Mdm2 in growth signaling and cancer. Growth Factors 23: 183-192, 2005.

43. Mayo LD and Donner DB: The PTEN, Mdm2, p53 tumor suppressor-oncoprotein network. Trends Biochem Sci 27: 462-467, 2002.

44. Kirch HC, Flaswinkel S, Rumpf H, Brockmann D and Esche H: Expression of human p53 requires synergistic activation of transcription from the p53 promoter by AP-1, NF-kappaB and Myc/Max. Oncogene 18: 2728-2738, 1999.

45. Zheng T, Meng X, Wang J, et al: PTEN- and p53-mediated apoptosis and cell cycle arrest by FTY720 in gastric cancer cells and nude mice. J Cell Biochem 111: 218-228, 2010.

46. Mayo LD, Dixon JE, Durden DL, Tonks NK and Donner DB: PTEN protects p53 from Mdm2 and sensitizes cancer cells to chemotherapy. J Biol Chem 277: 5484-5489, 2002

47. Sen P, Mukherjee S, Ray D and Raha S: Involvement of the Akt/PKB signaling pathway with disease processes. Mol Cell Biochem 253: 241-246, 2003

48. Zhou BP, Liao Y, Xia W, Zou Y, Spohn B and Hung MC: HER-2/neu induces p53 ubiquitination via Akt-mediated MDM2 phosphorylation. Nat Cell Biol 3: 973-982, 2001.

49. Li AG, Piluso LG, Cai X, Wei G, Sellers WR and Liu X: Mechanistic insights into maintenance of high p53 acetylation by PTEN. Mol Cell 23: 575-587, 2006.

50. Quevedo C, Kaplan DR and Derry WB: AKT-1 regulates DNA-damage-induced germline apoptosis in C.elegans. Curr Biol 17: 286-292, 2007.

51. Ming M and He YY: PTEN in DNA damage repair. Cancer Lett 319: 125-129, 2012.

52. Bonavida B and Baritaki S: The novel role of Yin Yang 1 in the regulation of epithelial to mesenchymal transition in cancer via

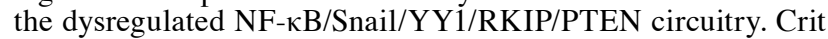
Rev Oncog 16: 211-226, 2011.

53. Vu BT and Vassilev L: Small-molecule inhibitors of the p53-MDM2 interaction. Curr Top Microbiol Immunol 348: 151-172, 2011.
54. Bixby D, Kujawski L, Wang S and Malek SN: The pre-clinical development of MDM2 inhibitors in chronic lymphocytic leukemia uncovers a central role for p53 status in sensitivity to MDM2 inhibitor-mediated apoptosis. Cell Cycle 7: 971-979, 2008.

55. Amodio N, Scrima M, Palaia L, et al: Oncogenic role of the E3 ubiquitin ligase NEDD4-1, a PTEN negative regulator, in nonsmall-cell lung carcinomas. Am J Pathol 177: 2622-2634, 2010.

56. Valiente M, Andrés-Pons A, Gomar B, et al: Binding of PTEN to specific PDZ domains contributes to PTEN protein stability and phosphorylation by microtubule-associated serine/threonine kinases. J Biol Chem 280: 28936-28943, 2005.

57. Torres $\mathrm{J}$ and Pulido R: The tumor suppressor PTEN is phosphorylated by the protein kinase CK2 at its $\mathrm{C}$ terminus. Implications for PTEN stability to proteasome-mediated degradation. J Biol Chem 276: 993-998, 2001.

58. Wu W, Wang X, Zhang W, et al: Zinc-induced PTEN protein degradation through the proteasome pathway in human airway epithelial cells. J Biol Chem 278: 28258-28263, 2003.

59. Li Y, Ozaki T, Kikuchi H, Yamamoto H, Ohira $M$ and Nakagawara A: A novel HECT-type E3 ubiquitin protein ligase NEDL1 enhances the p53-mediated apoptotic cell death in its catalytic activity-independent manner. Oncogene 27: 3700-3709, 2008.

60. Shinada K, Tsukiyama T, Sho T, Okumura F, Asaka M and Hatakeyama S: RNF43 interacts with NEDL1 and regulates p53-mediated transcription. Biochem Biophys Res Commun 404: 143-147, 2011.

61. Barata JT: The impact of PTEN regulation by CK2 on PI3Kdependent signaling and leukemia cell survival. Adv Enzyme Regul 51: 37-49, 2011.

62. Kang JY, Kim JJ, Jang SY and Bae YS: The p53-p21(Cip1/ WAF1) pathway is necessary for cellular senescence induced by the inhibition of protein kinase CKII in human colon cancer cells. Mol Cells 28: 489-494, 2009.

63. Marsh DJ, Kum JB, Lunetta KL, et al: PTEN mutation spectrum and genotype-phenotype correlations in Bannayan-RileyRuvalcaba syndrome suggest a single entity with Cowden syndrome. Hum Mol Genet 8: 1461-1472, 1999.

64. Marsh DJ, Coulon V, Lunetta KL, et al: Mutation spectrum and genotype-phenotype analyses in Cowden disease and BannayanZonana syndrome, two hamartoma syndromes with germline PTEN mutation. Hum Mol Genet 7: 507-515, 1998.

65. Zbuk KM and Eng C: Hamartomatous polyposis syndromes. Nat Clin Pract Gastroenterol Hepatol 4: 492-502, 2007.

66. Agrawal S, Pilarski R and Eng C: Different splicing defects lead to differential effects downstream of the lipid and protein phosphatase activities of PTEN. Hum Mol Genet 14: 2459-2468, 2005.

67. Teresi RE, Zbuk KM, Pezzolesi MG, Waite KA and Eng C: Cowden syndrome-affected patients with PTEN promoter mutations demonstrate abnormal protein translation. Am J Hum Genet 81: 756-767, 2007.

68. Han SY, Kato H, Kato S, et al: Functional evaluation of PTEN missense mutations using in vitro phosphoinositide phosphatase assay. Cancer Res 60: 3147-3151, 2000.

69. Kato H, Kato S, Kumabe T, et al: Functional evaluation of p53 and PTEN gene mutations in gliomas. Clin Cancer Res 6: 3937-3943, 2000

70. Georgescu MM, Kirsch KH, Kaloudis P, Yang H, Pavletich NP and Hanafusa $\mathrm{H}$ : Stabilization and productive positioning roles of the C2 domain of PTEN tumor suppressor. Cancer Res 60: 7033-7038, 2000 .

71. Trotman LC, Wang X, Alimonti A, et al: Ubiquitination regulates PTEN nuclear import and tumor suppression. Cell 128: 141-156, 2007.

72. Bonneau D and Longy M: Mutations of the human PTEN gene. Hum Mutat 16: 109-122, 2000.

73. Keniry M and Parsons R: The role of PTEN signaling perturbations in cancer and in targeted therapy. Oncogene 27: 5477-5485, 2008

74. Simpson L and Parsons R: PTEN: life as a tumor suppressor. Exp Cell Res 264: 29-41, 2001.

75. Carracedo A, Alimonti A and Pandolfi PP: PTEN level in tumor suppression: how much is too little? Cancer Res 71: 629-633, 2011.

76. Alimonti A, Carracedo A, Clohessy JG, et al: Subtle variations in Pten dose determine cancer susceptibility. Nat Genet 42: 454-458, 2010.

77. Xie CC, Lu L, Sun J, et al: Germ-line sequence variants of PTEN do not have an important role in hereditary and non-hereditary prostate cancer susceptibility. J Hum Genet 56: 496-502, 2011. 
78. Riegert-Johnson DL, Gleeson FC, Roberts M, Tholen K, Youngborg L, Bullock $M$ and Boardman LA: Cancer and Lhermitte-Duclos disease are common in Cowden syndrome patients. Hered Cancer Clin Pract 8: 6, 2010.

79. Ali E, Athanasopoulos PG, Forouhi P and Malata CM: Cowden syndrome and reconstructive breast surgery: case reports and review of the literature. J Plast Reconstr Aesthet Surg 64: 545-549, 2011.
80. Zhang G, Yang P, Guo P, Miele L, Sarkar FH, Wang Z and Zhou Q: Unraveling the mystery of cancer metabolism in the genesis of tumor-initiating cells and development of cancer. Biochim Biophys Acta 1836: 49-59, 2013.

81. Dean JL and Knudsen KE: The role of tumor suppressor dysregulation in prostate cancer progression. Curr Drug Targets 14: 460-471, 2013. 